Musées, Patrimoine et Culture scientifiques et techniques

$170 \mid 2017$

mars-avril 2017

\title{
TIME, un outil de visite innovant au service de tous
}

Virginie Duede

\section{OpenEdition \\ Journals}

Édition électronique

URL : http://journals.openedition.org/ocim/1753

DOI : $10.4000 /$ ocim. 1753

ISSN : 2108-646X

Éditeur

OCIM

Édition imprimée

Date de publication : 1 mars 2017

Pagination : 18-22

ISSN : 0994-1908

Référence électronique

Virginie Duede, "TIME, un outil de visite innovant au service de tous », La Lettre de l'OCIM [En ligne], 170 | 2017, mis en ligne le 01 mars 2018, consulté le 30 avril 2019. URL : http:// journals.openedition.org/ocim/1753; DOI : 10.4000/ocim.1753

Ce document a été généré automatiquement le 30 avril 2019.

Tous droits réservés 


\section{TIME, un outil de visite innovant au service de tous}

\section{Virginie Duede}

Le musée des Maisons comtoises de Nancray

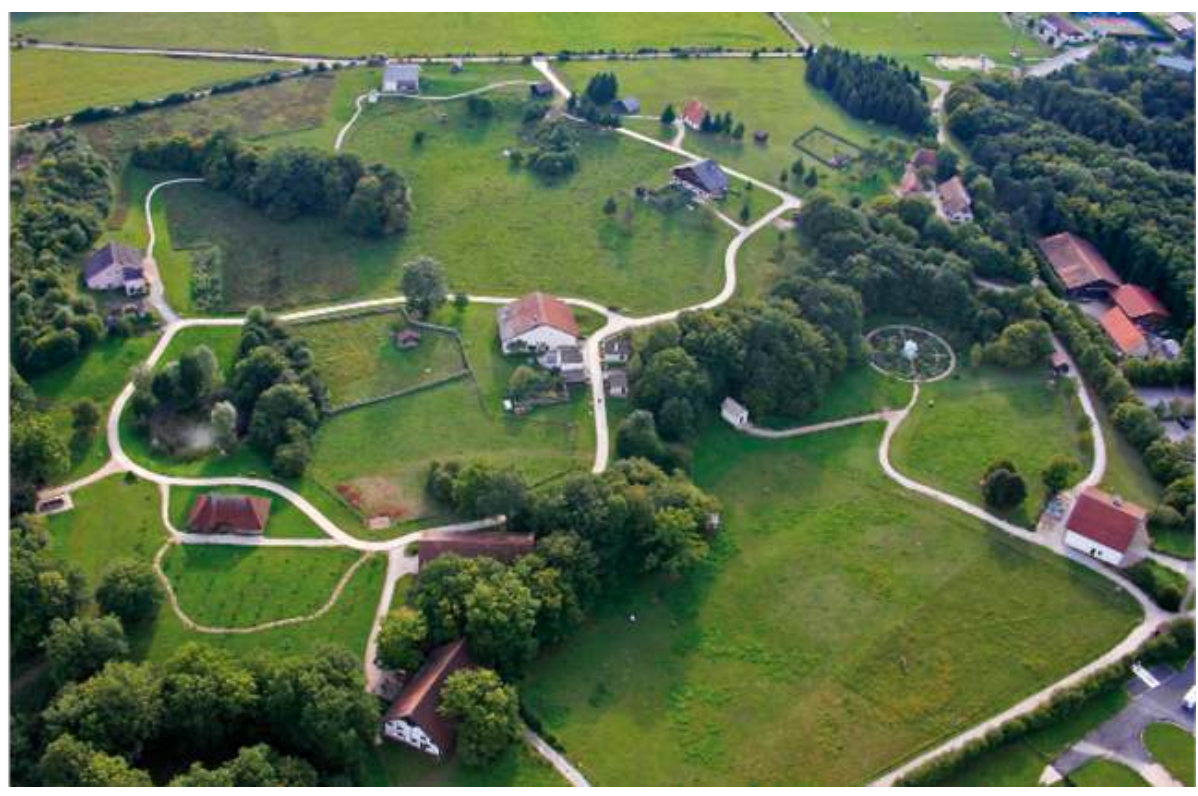

(C) Musée des Maisons comtoises

\section{Musée de plein air et accessibilité ?}

Le musée des Maisons comtoises figure parmi les cinq musées de plein air de France. Le principe de ces musées est la sauvegarde de maisons traditionnelles, avec l'ensemble de leur mobilier, des objets, de l'outillage, par leur démontage et leur reconstruction dans un même parc. Créé en 1984 à Nancray (Doubs), le musée de plein air des Maisons comtoises s'étend sur un site vallonné d'une quinzaine d'hectares, sur lequel est implanté une 
trentaine d'édifices. Ce bâti traditionnel comtois des XVIIe, XVIIIe et XIXe siècles est accompagné de potagers, jardins thématiques et pâtures. Les intérieurs présentent une évolution des modes de vie de 1750 à 1950 et cinq granges ont été transformées en salles d'exposition.

2 Les questions d'accessibilité du musée se sont posées à partir de 2007 suite à un nouveau Projet Scientifique et Culturel (PSC) initié et rédigé par Marie Spinelli-Flesch, conservatrice en chef du patrimoine et directrice du musée de 2005 à 2015. Dans ce projet la dimension sociale en tant que pilier du Développement Durable n'est pas négligée et sera même le levier pour les questions d'accessibilité de ce lieu atypique. Cet axe du projet débute à partir de 2009 avec la mise en place de plusieurs actions facilitant la découverte du musée aux personnes en situation de handicap.

En 2010, se pose la question de l'accessibilité des personnes à mobilité réduite avec deux difficultés majeures auxquelles répondre : comment se déplacer dans un parc de quinze hectares avec de fortes pentes, et comment rendre compte de la vie des franc-comtois du XVIIe siècle au XXe siècle à partir de leur habitat, sans les altérer ? Seule une solution de substitution via une visite virtuelle des pièces et des maisons-collections non accessibles était possible. En 2010 une telle offre n'existait pas encore. C'est une entreprise de Morteau (Doubs), spécialisée dans les panoramas à $360^{\circ}$ pour les sites Internet, qui a accepté de travailler sur le sujet. Après plusieurs mois de recherches et de développement, une application de visite virtuelle du musée est créée en 2012.

Pour les phases de test de ce projet, le travail a été réalisé en collaboration avec des résidents de la maison de retraite voisine du musée. De ce travail de concertation est née l'idée de constituer un comité de pilotage regroupant des personnes en situation de handicap, dont la mission est d'accompagner le musée dans sa démarche d'accessibilité dans le cadre de la loi du 11 février 2005 sur l'égalité des droits et des chances. Cette démarche participative et ce projet innovant valent au musée d'être récompensé en 2013 par le ministère de la Culture et de la Communication du prix "Patrimoine pour tous". Ce prix est un levier, car il accélère la prise de conscience de l'ensemble des agents et des membres du conseil syndical ${ }^{1}$ sur l'importance de rendre accessible au plus grand nombre le musée. L'accessibilité pour tous devient une des actions prioritaires du musée de 2013 à 2015.

La ferme du Haut-Doubs des Arces de Morteau bâtie en 1784 : le musée présente un certain nombre de maisons caractéristiques de Franche-Comté $\odot$ Musée des Maisons comtoises

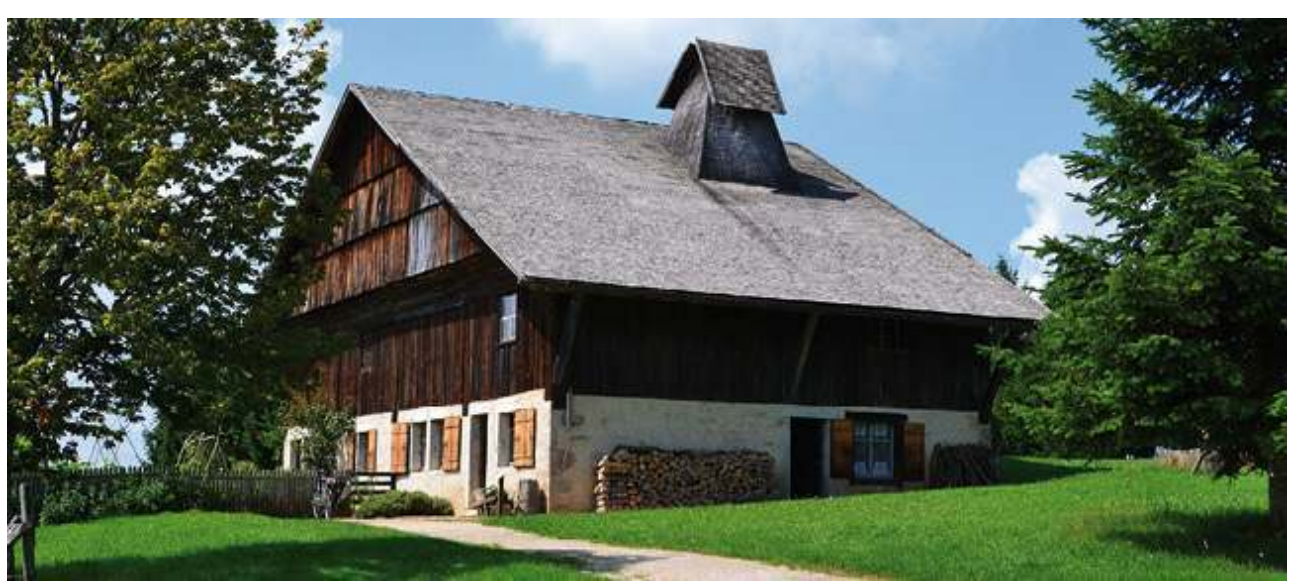

(c) Musée des Maisons comtoises 
5 Ainsi, du travail du comité de pilotage ressort l'objectif de prioriser l'accessibilité aux contenus culturels par rapport à celui de l'accessibilité physique, car la demande émanant des usagers est d'abord de pouvoir partager un moment en famille ou entre amis. Pour cela, il est nécessaire que chacun ait accès aux contenus scientifiques relatifs aux collections, à tout moment et pas seulement sur réservation. Par souci d'équité et d'égalité, le musée travaille alors sur des projets de visites pour tous. Or, il s'avère qu'il n'était pas possible de former l'ensemble des agents d'accueil et de médiation du musée à la Langue des Signes Françaises, au Langage Parlé Complété et aux visites tactiles auxquelles s'ajoutent les difficultés à s'exprimer dans une langue étrangère. Ce dispositif aurait été trop coûteux et peu efficient, puisque le personnel ne serait pas amené à pratiquer assez souvent, le musée recevant 45000 visiteurs sur une période d'ouverture de sept mois. Fort de cette première expérience de visite virtuelle, le musée s'est orienté vers une solution de substitution intégrant les nouvelles technologies.

Les visiteurs peuvent découvrir l'intérieur des habitations comme cette cuisine muséographiée années 1950 dans la ferme des Vosges saônoises, La Poiselière, datant de 1761. () Musée des Maisons comtoises

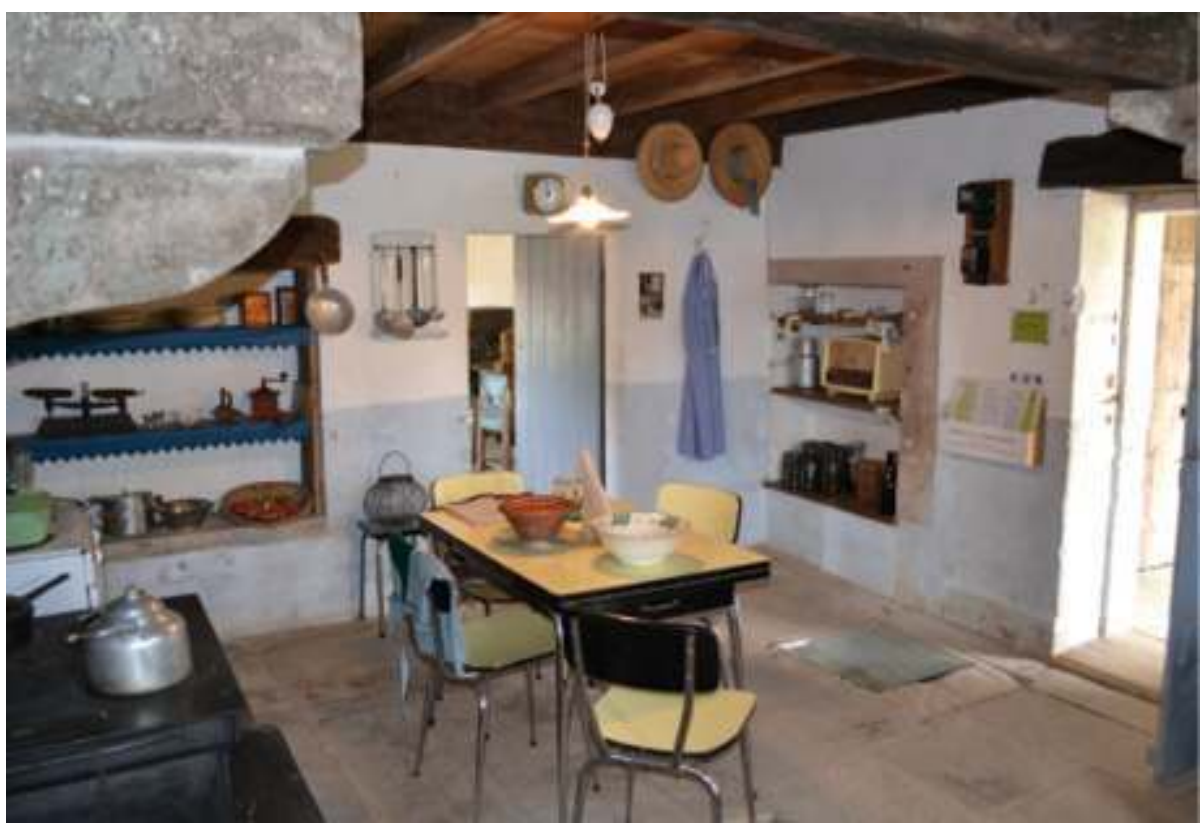

(c) Musée des Maisons comtoises

6 Ayant connaissance de systèmes de visites téléchargeables, ou de systèmes utilisant la technologie du flash code, nous cherchions un autre procédé ne pénalisant pas les visiteurs par l'utilisation de leur forfait Internet ou n'ayant pas téléchargé au préalable l'application de visite (seule une personne sur quatre possédant un smartphone qui télécharge des applications). Cette solution n'existait pas encore. Parallèlement, en 2013, une entreprise de Besançon travaillait à la recherche et au développement d'une innovation technologique, simple d'usage, sans téléchargement, permettant l'accès d'un grand nombre d'usagers à des gigas de données sur leurs propres appareils mobiles (smartphones et tablettes), sans contrainte de distance.

7 D'une rencontre fin 2013, est née l'idée de la création d'un outil de visite de substitution permettant aux personnes en situation de handicap auditif et visuel d'avoir accès à différentes visites audio ou vidéo sur leur smartphones et aussi des visites pour les 
personnes anglophones et germanophones sur l'ensemble des quinze hectares du musée. Une fois encore, l'innovation technologique était la solution aux besoins du musée en terme d'accessibilité. C'est ainsi qu'est né le projet TIME. Conçu comme une aventure immersive dans le temps et l'espace, TIME (Time Machine Museum Experience) est aussi un clin d'œil à la Franche-Comté, terre d'utopie et d'innovation reconnue pour ses savoirfaire industriels, en matière d'horlogerie notamment.

\section{De l'accessibilité au projet TIME}

8 L'accessibilité est le principe fondateur de ce projet, dont l'un des objectifs est de transmettre des connaissances sur le patrimoine régional à tous, sans discrimination, renforçant la place du musée en tant qu'acteur de référence en région, par la qualité de ses offres culturelles.

9 Après huit mois de recherches de partenaires financiers, le projet a évolué de l'accessibilité à un outil de visite pour tous. C'est ainsi qu'à partir de juin 2014, le travail a porté sur la mise en œuvre d'un outil innovant de visite, qui soit tout à la fois connecté au territoire régional et au service de tous les publics : familles, enfants, seniors, personnes en situation de handicap et public étranger. TIME est un outil de diffusion de contenus scientifiques et culturels, qui donne accès à des visites guidées (en anglais, allemand, langue des signes, audio-description...) et à un service ludique en 3D destiné aux plus jeunes. Pour la réalisation de ce projet, le musée a reçu le soutien de la Fondation de France, en répondant à l'appel à projet "Accès de tous à tous", de la région FrancheComté, dans le cadre de l'appel à projet "Innovation touristique en Franche-Comté" et enfin de l'appel à projet "Projet service numérique culturel innovant 2014" du ministère de la Culture et de la Communication.

10 Les objectifs du projet étaient :

11 - ouvrir et connecter directement le discours du musée à son territoire ;

12 - faire connaître aux différents publics les richesses de la région et leur faire comprendre comment les activités d'aujourd'hui puisent leurs racines dans celles d'hier et en sont les prolongements ;

13 - s'inscrire dans une politique d'accessibilité ambitieuse, en favorisant l'accès des différents publics aux contenus culturels et scientifiques du musée ;

14 - s'intégrer dans la politique sociale du musée en donnant accès à des contenus scientifiques et culturels, gratuitement à tous, soit sur les smartphones ou les tablettes des visiteurs soit en prêtant le matériel.

\section{Le système}

15 La conception de l'outil de visite a nécessité huit mois de recherches et développements et son installation deux mois. Le musée est équipé du dispositif Praedi-X. Il s'agit d'un système de huit stations, réparties dans le parc de quinze hectares, composées de serveurs et d'antennes créant un réseau sans fil, capable de diffuser des contenus audio, vidéo, texte dans l'ensemble des maisons-collections. Les contenus sont diffusés dans de bonnes conditions de qualité à travers un réseau sans fil haut débit. La lecture des contenus s'effectue de manière instantanée, sans temps de latence tout en utilisant des 
médias vidéo, audio, image de haute qualité, combinés avec des informations de géolocalisation. L'absence de téléchargement et de connexion à Internet, très énergivore a pour conséquence une faible sollicitation des équipements des visiteurs. La diffusion est compatible avec tous les terminaux mobiles actuels et futurs disposant d'un navigateur Internet et d'une connectivité Wifi. Les dispositifs fonctionnent en lien avec un système de gestion centralisé permettant la synchronisation des contenus et des scénarii. L'ensemble des interactions entre les terminaux mobiles et le dispositif Praedi-X remonte au système de gestion centralisé afin de fournir des statistiques détaillées sur les contenus visualisés et les actions effectuées.

La page d'accueil de l'outil de diffusion TIME

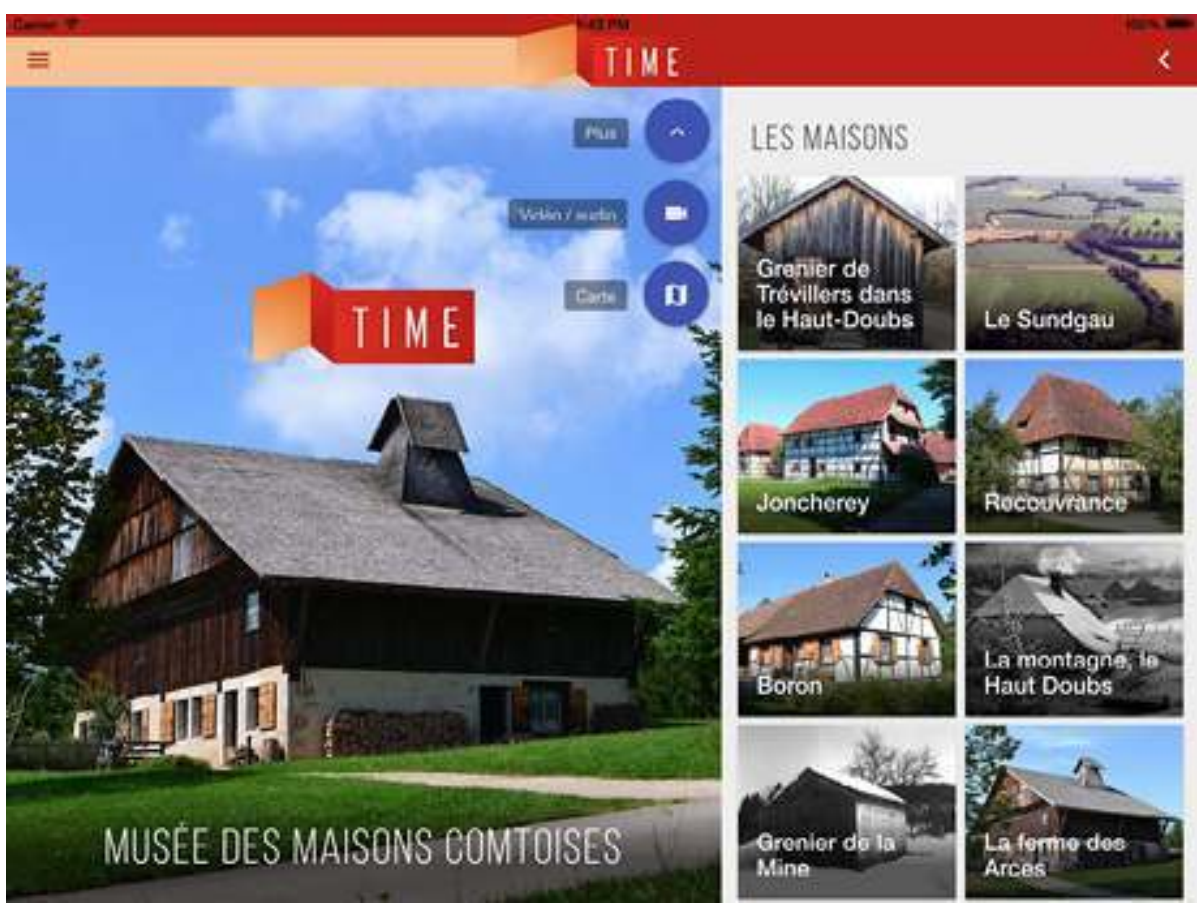

(c) Musée des Maisons comtoises

16 L'accès aux visites est simple. Lors de son arrivée au musée, il suffit d'activer le Wifi de son smartphone ou de sa tablette. Le terminal reconnaît le réseau Wifi TIME, il ne reste plus alors qu'à se connecter et la page d'accueil de TIME s'ouvre automatiquement. La navigation est simple, puisqu'elle reprend les systèmes de boutons couramment utilisés. Le visiteur peut choisir sa préférence de visite, entre toutes celles proposées : français, anglais, allemand, sous-titrage, Langue des Signes Française, Langage Parlé Complété, Audio-description. L'accès à la connaissance est donc gratuit pour tous les visiteurs. Pour ceux qui ne sont pas équipés, le musée prête des tablettes. L'objectif prioritaire du projet TIME est d'offrir grâce à la technologie ce que l'équipe de médiation ne peut pas transmettre aux visiteurs, de garantir à tous la même qualité de service.

\section{Les contenus médias}

17 Élaborés avec et pour les publics, les contenus sont adaptés à chacun. Leur rédaction a été réalisée en interne par l'équipe scientifique du musée sur une période de cinq mois. Pour les visites à destination des personnes en situation de handicap, le personnel du musée 
s'est fait aider par le comité de pilotage du musée afin que ces visites répondent le mieux possible à leurs besoins. La visite générale est composée de 68 vidéos présentant les différentes micro-régions de la Franche-Comté, les maisons-collections et les pièces d'habitation. Elle est enrichie de 72 bonus, présentant en quelques lignes des objets de collection exposés et des images d'archives de la Franche-Comté. Cette visite permet non seulement une compréhension du site dans son ensemble, mais elle donne en plus une lecture de la région grâce aux contenus numériques d'une soixantaine de partenaires. Ils permettent d'élargir la visite à l'ensemble de la Franche-Comté.

18 Lors de leur visite, les visiteurs ont la possibilité de découvrir des lieux, des savoir-faire, des artisans, des entreprises, des filières en lien direct avec ce qu'ils voient au musée. Par exemple pendant la visite de la ferme des Bouchoux, en plus de la vidéo de présentation générale de la ferme, il est possible de lire des fiches bonus sur les charpentes sur colonne, les couvertures en bois, le tavaillon ${ }^{2}$ et sur des bonus partenaires comme ceux de l'entreprise Simonin et la Maison du parc naturel régional du Haut-Jura (39). Dans le premier espace de la maison, le corridor, la vidéo explique la fonction de cette pièce et les différents objets s'y trouvant. Deux bonus proposent de découvrir deux objets en particulier, dont un tourné, un bout à cul (tabouret à pied tourné). Les bonus partenaires de cette pièce mettent en avant trois structures jurassiennes en lien avec ce thème : le musée du Jouet de Moirans-en-Montagne, l'entreprise Janod, fabricant de jouets et AsBois - Y'à pas le feu au lac, tourneurs sur bois et designers. Au travers de cette visite et des partenaires présentés, le visiteur peut faire le lien entre savoir-faire traditionnels et innovation industrielle actuelle. Elle permet de mettre en réseau un certain nombre d'établissements et de valoriser les richesses du territoire, tant sur les plans culturel économique, artisanal et industriel.

Le projet donne ainsi une lisibilité :

20 - au caractère agricole et industriel de la Franche-Comté ainsi qu'au tourisme qui s'y rapporte ;

21 - aux métiers présentés au musée et qui aujourd'hui sont soit restés les mêmes (savoirfaire en matière de couverture des maisons en bois ou en pierre par exemple), soit ont évolué sur le plan technologique (filière comté, lunetterie, verrerie, distillation, métallurgie, horlogerie et micro techniques) ou ont donné lieu à de nouveaux services à la population (nouveaux usages du cheval comtois).

Plusieurs types de visites sont disponibles:

23 - la visite en audio-description : réalisée en collaboration avec une audio-descriptrice, cette visite permet aux personnes en situation de handicap visuel de découvrir l'ensemble du musée et leur offre la possibilité de toucher certains objets ou détails d'architectures. Elle est constituée de 70 bandes son ;

24 - la visite jeune public : le musée a souhaité également présenter une visite pour le jeune public afin de répondre à l'évolution des pratiques culturelles des jeunes et favoriser l'échange transgénérationnel autour de la visite. Elle vient compléter les différents jeux de piste en libre accès en proposant aux visiteurs, une série de six petits jeux permettant de comprendre l'évolution des conditions de travail de la vie quotidienne entre 1750 et 1950. Il s'agit d'éveiller les utilisateurs à l'évolution de la condition de l'enfant de la fin de l'Ancien Régime à 1950 au travers d'épreuves. Les enfants, via la géolocalisation, doivent identifier des objets anachroniques, associer le bon objet à la bonne activité et trouver des objets de collection dans les présentations permanentes. 
TIME propose des jeux de piste pour découvrir la vie quotidienne dans les fermes.

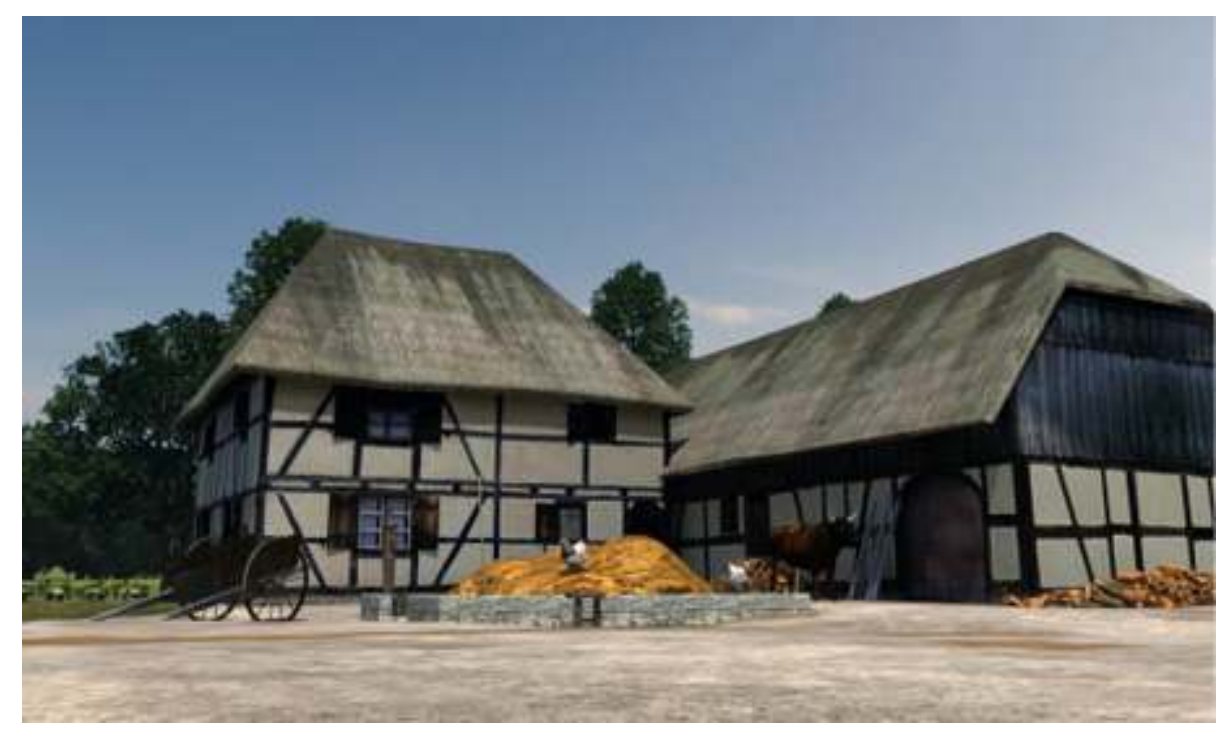

(c) Musée des Maisons comtoises

\section{La réception par les publics}

Pour mieux comprendre la perception des publics face à cet outil de visite, une présentation préalable de la politique de médiation du musée est nécessaire.

Le service des publics est composé de 9 personnes (6,6 ETP) pour une équipe de 30 personnes (22 ETP). L'offre culturelle se partage entre les ateliers pédagogiques, les stages pour les adultes, les manifestations et les offres aux familles. Cette dernière privilégie la médiation humaine favorisant la transmission par l'expérience et la discussion avec les visiteurs. Tous les jours, un programme d'une dizaine d'animations thématiques d'une vingtaine de minutes (parmi une centaine possible) est proposé aux visiteurs. Ces échanges se font sur des thèmes très variés : l'architecture, l'environnement, les savoirfaire, les jardins, la cuisine, l'histoire régionale...

Le musée est vécu par les visiteurs comme un lieu d'expériences multiples, accessibles à tous et pour se libérer des temps imposés. Fréquenté à $63 \%$ par des habitants de la région, le public vient chercher des moments de transmission et de convivialité en contact direct avec les médiateurs, dans un musée à ciel ouvert.

L'outil de visite TIME vient compléter cette offre, en permettant aux visiteurs un accès à d'autres contenus, avec une approche plus individuelle. Dès l'accueil, les avis du public divergent sur la pertinence de visiter ce lieu atypique avec son téléphone à la main, aussi TIME est présenté parmi un panel d'outils de médiation pour explorer le musée. Chacun est libre de son parcours et de sa façon d'aborder les collections et le parc et de se connecter ou non. 
TIME est adapté aux visiteurs en situation de handicap : cette vidéo permet ainsi une présentation du poêle de la ferme de Joncherey en Langue des Signes Française.

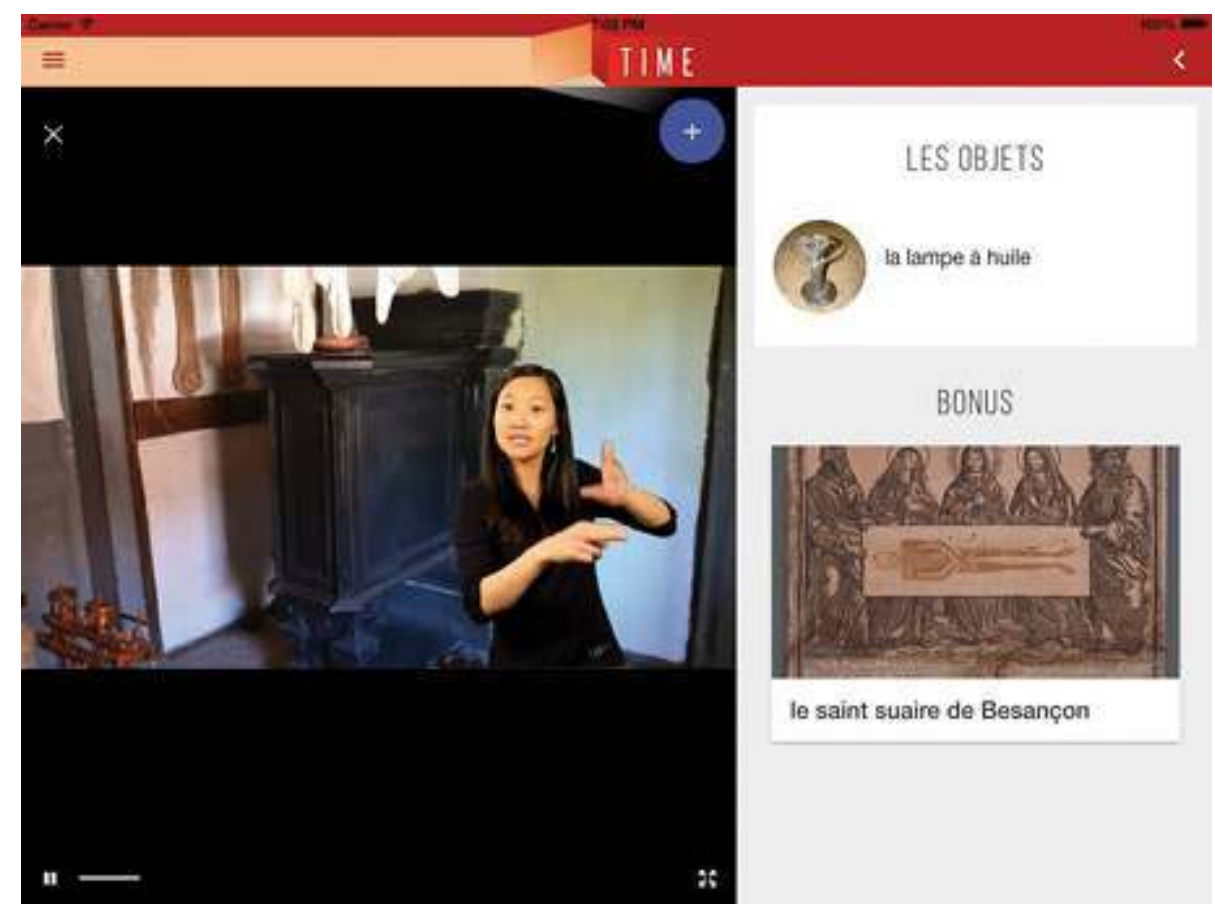

(C) Musée des Maisons comtoises

En fin de visite, les visiteurs étrangers et les visiteurs en situation de handicap sont enthousiastes de leurs visites, ils apprécient d'avoir eu accès à de nombreuses informations dans leur langue maternelle ou adaptées à leur handicap. Leur temps de visite s'est d'ailleurs allongé, ils restent désormais la journée au musée, contre deux heures auparavant. La fréquentation de ces publics à peu progressé. La part des visiteurs étrangers est passée de $3 \%$ à $4,5 \%$ et celle des visiteurs en situation de handicap progresse également lentement. Les primo-visiteurs sont très satisfaits, alternant renseignements sur les collections via TIME et via les médiateurs. Les familles sont les plus réfractaires à cette proposition de visiter le musée, argumentant que l'un des objectifs de cette sortie est de soustraire leur enfant à tout écran afin de partager un moment ensemble.

\section{Les perspectives de développement}

\section{Pour le musée}

30 Les perspectives de développement sont multiples : nouvelles langues, nouveaux bonus, nouveaux thèmes de visites (biodiversité, faune-flore, les jardins). Par ailleurs, le système permet une perspective de développement inattendue pour le musée.

31 L'étendue du musée (quinze hectares) rend difficile la surveillance des collections. Les serveurs et le système de transmission utilisés pour TIME peuvent être utilisés simultanément pour un système de vidéo surveillance dans l'ensemble du parc et des maisons-collections.

32 Le développement de cet outil peut également entraîner : 
- la création de nouveaux bonus partenaires illustrant l'évolution des savoir-faire ;

- l'intégration et la présentation de davantage de documents d'archives et de documents iconographiques illustrant, contextualisant et enrichissant les collections présentées ;

- l'insertion de films en bonus, permettant de mieux comprendre les gestes des savoirfaire (tisserand, bourrelier).

\section{Pour d'autres sites}

Le système est transposable partout. Par l'utilisation des terminaux des usagers et la possibilité de contenus illimités, cet outil offre de belles perspectives dans le cadre de l'accessibilité à tous ou pour la valorisation et la diffusion de sources documentaires, iconographiques non accessibles aux visiteurs. De plus, la technologie employée permet une utilisation dans des conditions particulières voire extrêmes : en juin 2015, la température relevée dans un des greniers où se trouve l'une des bornes était de $45^{\circ} \mathrm{C}$. Le système de diffusion de contenus ne nécessite pas de câblage ce qui facilite sa mise en place, notamment dans des lieux patrimoniaux.

7 Le confort de visite est appréciable, puisque les médias restent sur les serveurs. Un des autres avantages de ce système est qu'il n'impose aucune contrainte de taille de fichiers.

Bien que délicate la question de la nocivité des émissions d'ondes électromagnétiques n'est pas à négliger dans un tel projet. L'installation utilise des antennes WiFi en $2.4 \mathrm{Ghz}$ avec une puissance de $100 \mathrm{~mW} /$ antenne. Chaque antenne (directionnelle à $120^{\circ}$ ) couvre une zone prédéfinie sans chevauchement (ou avec un chevauchement très mineur). Des mesures, dans le parc du musée, révèlent une activité cumulée de $0.05 \mathrm{v} / \mathrm{m}$. Une des plusvalue de ce dispositif de visite est son outil statistique intégré performant qui permet de comptabiliser les comportements multimédia des visiteurs et donc de multiples analyses.

Cet outil de visite innovant est né de la démarche d'accessibilité entreprise par le musée dès 2009, dans le cadre de l'axe équité sociale de son Projet Scientifique et Culturel et des obligations liées à la loi du 11 février 2005. Au départ, cette loi a été vue et vécue comme une contrainte face à l'ampleur de la tâche, ce n'est plus le cas aujourd'hui. Au contraire cette obligation légale nous a obligés à avoir une lecture critique sur nos cheminements, l'agencement de nos espaces, les choix muséographiques et nos méthodes de médiation. Grâce au travail de concertation avec des usagers et de nouvelles possibilités technologiques, le musée de plein air des Maisons comtoises a su renouveler son offre pour tous les publics et ouvrir son discours à son territoire. Cette démarche a d'ailleurs été récompensée par l'obtention des quatre labels Tourisme et Handicap et renforce son rôle en tant que musée de société, acteur social sur son territoire.

\section{NOTES}

1. Le musée est géré par un Syndicat mixte composé à part égale du Conseil départemental du Doubs et de la Communauté d'Agglomération du Grand Besançon. 
2. Le tavaillon est une tuile de sapin blanc ou d'épicéa fendu, il se présente sous forme d'une petite planchette mesurant, selon les lieux et les époques, 24 à $48 \mathrm{~cm}$ de long, 4 à $19 \mathrm{~cm}$ de large et 0,1 à $1,1 \mathrm{~cm}$ d'épaisseur.

\section{RÉSUMÉS}

Lié à l'origine à la démarche d'accessibilité mise en place dans le cadre du Projet Scientifique et Culturel du musée des Maisons comtoises de Nancray, cet outil de diffusion d'informations scientifiques et culturelles s'est finalement développé en direction de l'ensemble des publics, permettant un accès différent à des contenus non diffusés par l'offre classique de médiation ou en direction des visiteurs avec lesquels les médiateurs ont du mal à communiquer.

INDEX

Mots-clés : accessibilité, public, médiation

\section{AUTEUR}

\section{VIRGINIE DUEDE}

directrice du musée des Maisons comtoises à Nancray

virginieduede@maisons-comtoises.org 